\title{
Wave propagation in nonlinear photonic band-gap materials
}

\author{
Qiming Li \\ Ames Laboratory-United States Department of Energy and Department of Physics and Astronomy, \\ Iowa State University, Ames, Iowa 50011 \\ C. T. Chan \\ Ames Laboratory-United States Department of Energy and Department of Physics and Astronomy, \\ Iowa State University, Ames, Iowa 50011 \\ and Physics Department, Hong Kong University of Science and Technology, Clear Water Bay, Hong Kong
}

K. M. Ho and C. M. Soukoulis

Ames Laboratory-United States Department of Energy and Department of Physics and Astronomy, Iowa State University, Ames, Iowa 50011

(Received 19 December 1995)

\begin{abstract}
We study theoretically the propagation of electromagnetic waves through periodic structures consistent of layered materials with an intensity-dependent dielectric constant. We find the transmission properties to be strongly modulated by both frequency and intensity in the presence of nonlinearity. The transmission diagram in the frequency versus amplitude plane exhibits distinctive features depending upon whether the Kerr coefficient is positive or negative. These features, though complicated, can be understood through the analysis of stable periodic orbits of the corresponding nonlinear mapping. These systems exhibit bistability and multistability most strongly near the upper band edges and between the basins of stable periodic orbits. Resonance transmissions via soliton formation are analyzed through a simple mechanical analogy. We also discuss the switching threshold and the feasibility of making a switch utilizing such a structure. [S0163-1829(96)01324-0]
\end{abstract}

\section{INTRODUCTION}

When dielectric materials are arranged periodically in certain ways, light at some frequencies is forbidden to propagate in any direction. The utilization of this remarkable property of the recently discovered photonic band-gap (PBG) crystals for device applications is currently under intensive investigations. ${ }^{1}$ Almost all the existing work focuses on the linear regime in which the dielectric constant is independent of the field strength. It is well known that the presence of optical nonlinearity in a system leads to a much richer and more complex response to radiation. Examples include the formation of solitons in optical fibers ${ }^{2}$ and optical bistability in nonlinear Fabry-Perot etalon. ${ }^{3,4}$ These phenomena have important potential technological applications in high-speed optical communication systems and in ultrafast optical switches. ${ }^{5}$ We believe that incorporating nonlinearity in PBG materials may prove to be equally fruitful. However, before attacking the full three-dimensional problem, which is much more challenging, we need a better understanding of the global picture of the propagation of electromagnetic (EM) waves in nonlinear media by using a simpler structure. Such studies in low dimensions may also prove useful for engineering structures that have very low thresholds for switching.

In this paper, we investigate theoretically the nonlinear response of wave propagation in a one-dimensional analog of the PBG material: a multilayered structure consisting of alternating dielectric material. These structures, commonly known as Bragg reflectors, exhibit forbidden regions of EM wave transmission in the layering direction known as stop bands. The possibility of achieving optical bistable switching in these distributed feedback structures was first pointed out by Winful et al. ${ }^{6}$ and demonstrated experimentally with a GaAs/AlAs periodic structure. ${ }^{7}$ In essence, the bistable response results from the modulation of the transmission by an intensity-dependent phase shift, ${ }^{5}$ in much the same way as occurred in the traditional nonlinear Fabry-Perot etalon. Additional EM wave transmission modes were discovered within the stop bands in a numerical study ${ }^{8}$ of the wave propagation in nonlinear superlattice structures. These modes are localized solutions, known as "gap solitons." Under appropriate conditions, they can couple with the incident wave to achieve resonant transmission. ${ }^{8,9}$ Gap solitons can also exist in higher dimensions. ${ }^{10}$ Recently, the propagation of ultrashort pulses in nonlinear superlattices ${ }^{11}$ has also been investigated. Due to the nonlinear nature of the problem, the response to a pulse cannot be obtained from the response to plane-waves by superposition. Even for plane-wave input, a global picture of the optical response, has not been presented yet. Much more is known about the response of electrons in a nonlinear lattice. ${ }^{12,13}$ In this case, the periodic modulation of the nonlinear media is provided by the discrete nature of the lattice. A recent attempt ${ }^{14}$ to formulate the transmission problem of EM waves through nonlinear layered media neglected the inherent difference between EM waves and electronic waves, and the phase diagram obtained is thus only appropriate to electrons. Two features of the present study differ from the previous studies, ${ }^{12,13}$ (1) the effectiveness of the nonlinearity is strongly modified by the frequency, due to the different behavior of the Maxwell and Schrödinger equation, and (2) the modulation of dispersion relation by super- 
lattice breaks the symmetry between positive and negative nonlinear media and makes the nonlinear response to the EM wave much richer and more interesting.

In what follows, we shall formulate the problem of the EM transmission in nonlinear layered structures and investigate in detail its transmission characteristics. In contrast to the electronic systems ${ }^{12,13}$ studied previously, the strength of the nonlinearity depends upon both the frequency and the intensity of the incident wave. Thus, the transmission diagram is quite different from that of the electronic response in a nonlinear lattice. This is especially true for nonlinear media with negative Kerr coefficients, where the strong modification of the nonlinearity by frequency, together with the physical boundary condition, conspires to make the transmission diagram more complex and interesting. However, we shall show that as complex as it may appear, the overall features can be understood through analysis of stable periodic orbits of the nonlinear mapping and analysis of various bounds of the nonlinear difference and the corresponding differential equations. We also present a simple and intuitive picture of the soliton and soliton trains based upon a mechanical analogy. We investigate the bistability and multistability in different regions of the parameter space and discuss their potential use as switches.

Our analysis is based on the steady state response of local Kerr media to plane-wave input. The effect of absorption, nonlocal nonlinearity, and possible dynamic and transverse instability are not included. These effects could be important for the operation of switching devices. In semiconductors, the diffusive nature of the nonlinearity counteracts the transverse instability and makes the plane-wave analysis more relevant to experiment.

This paper is organized as follows. The theoretical formulation of the wave transmission in a nonlinear superlattice shall be presented in Sec. II, along with some discussions on the general symmetry properties of the resultant difference equation. The transmission diagrams from the numerical integration of the difference equation will be presented in Sec. III. Also presented in Sec. III is some theoretical understanding of these rich and complex features, including the presence of gap-soliton solutions. The bistable and multistable response will be discussed in Sec. V, and we conclude in Sec. VI.

\section{FORMULATION OF THE PROBLEM}

Consider a structure of $2 \mathrm{~N}$ alternating layers of two dielectrics with linear dielectric constant $\epsilon_{0 a}$ and $\epsilon_{0 b}$ and thickness $a$ and $b$, respectively. The nonlinearity of one of the dielectrics, say media $A$, is much larger than the other, so we can consider the later as linear. The steady state plane-wave transmission problem can be formulated as follows. A planewave of wave vector $k$ is normally incident on the structure from the left. A fraction of the wave is transmitted through the structure and exits from the right and the rest is reflected back. In this study, we concentrate on the stationary response only and shall not deal with the transient behavior and possible chaotic dynamics that have been discussed in previous studies. ${ }^{9}$ Transverse effects have also been neglected.

The EM waves outside the nonlinear structure are described by

$$
E(x, t)= \begin{cases}E_{0} e^{i(k x-\omega t)}+E_{r} e^{-i(k x+\omega t)} & \text { for } x<0 \\ E_{t} e^{i(k x-\omega t)} & \text { for } x>N,\end{cases}
$$

where $k=\omega / c, \omega$ is the optical frequency, and $c$ is the vacuum speed of the light. Within the structure, the propagation of a wave is governed by the Maxwell equations. The transmission coefficient is defined as

$$
T=\frac{\left|E_{t}\right|^{2}}{\left|E_{0}\right|^{2}}
$$

We are interested in the transmission characteristics for different frequencies and input or output intensities.

To obtain the transmission characteristics, one usually solves the nonlinear wave equation within each layer and then matches the waves with appropriate boundary conditions. ${ }^{4,8}$ Since the solution of the nonlinear equation is expressed as the inverse of a Jacobi elliptic integral, analytical calculation becomes nontransparent when the number of layers exceeds three. In this study, instead of taking this approach, we investigate the response of the system in the limit that the nonlinear layer is so thin, such that the field within it can be approximated as a constant. This is the case when the nonlinear layer thickness is small compared with the effective wavelength within it. Under this condition, the KronigPenney $\delta$-function model becomes adequate. Thus, the electric field obeys,

$$
\begin{aligned}
& \frac{d^{2} E(x)}{d x^{2}}+\frac{\epsilon \omega^{2}}{c^{2}} \sum_{n=1}^{N}\left[1+\lambda|E(x)|^{2}\right] E(x) \delta(x-n)+k^{2} E(x) \\
& \quad=0 .
\end{aligned}
$$

Within the linear layers, the wave consists of two planewaves traveling in opposite directions. Eliminating the waves in the linear media results in a difference equation in terms of the field at the nonlinear layers, $E_{n}$,

$$
E_{n+1}+E_{n-1}=\left[2 \cos k-\alpha k \sin k\left(1+\lambda\left|E_{n}\right|^{2}\right)\right] E_{n},
$$

where we have adopted the Kerr-type of local nonlinearity, $\epsilon_{a}=\epsilon_{0 a}+\lambda \alpha|E|^{2}$. The nonlinear Kerr coefficient is $\lambda \alpha$, where $\alpha=\epsilon_{0 a} \times a$. We have assumed the linear media are a vacuum $\left(\epsilon_{b}=1\right)$, and the distance between the neighboring nonlinear layers, $d=a+b$, is taken as one unit length. Equation (3) is valid when $d \ll \lambda \sqrt{\epsilon_{n}}$, but $\epsilon_{n} d \gg 1$. It happens that such a model captures most of the essential features of the nonlinear response of the superlattice structures and is simple enough to allow for analytical treatment under special conditions, as we shall see later.

Equation (4) has the property of conserving the energy flux $J=2 i\left(E_{n} E_{n+1}^{*}-E_{n}^{*} E_{n+1}\right)$. Moreover, it is invariant under a global gauge transformation $E_{n} \rightarrow e^{i \theta} E_{n}$. This means one can always take $E_{t}$ to be a real positive number. The strength of the nonlinearity can be absorbed into the field by a simple rescaling of the field $E \rightarrow E / \sqrt{|\lambda|}$. We shall vary the field strength, but keep $|\lambda|=1$. We also note that Eq. (4) has the same structure as the discretized version of the stationary nonlinear Schrödinger equation for the electron transmission problem, ${ }^{12,13}$

$$
\psi_{n+1}+\psi_{n-1}=-\Omega \psi_{n}-\lambda_{e}\left|\psi_{n}\right|^{2} \psi_{n}
$$




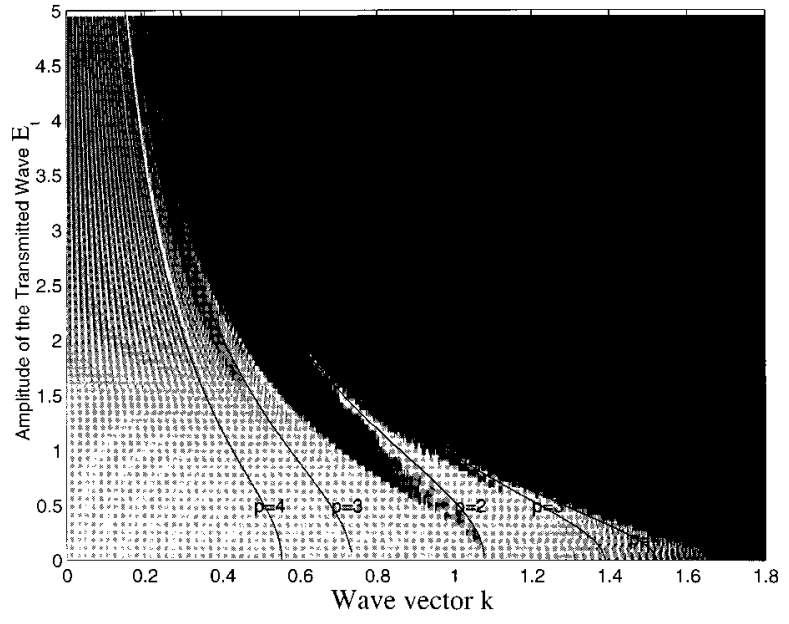

FIG. 1. The transmission diagram for a nonlinear superlattice with a positive Kerr coefficient showing the broadening of the instability regions (dark) and the developing of instabilities around the stable low-period orbits (shown as lines). $N=80$ and $\alpha=1$. Higher bands (not shown) show a similar behavior.

when we make the identification

$$
\begin{gathered}
\psi_{n} \leftrightarrow E_{n}, \\
\lambda_{e} \leftrightarrow \lambda \alpha k \sin k, \\
\Omega \equiv-2 \cos K \leftrightarrow-2 \cos k+\alpha k \sin k .
\end{gathered}
$$

It is clear that the effective nonlinearity is strongly modified by the wave vector (or frequency) in the EM case. The nonlinearity is much less effective in the long wave regime and at the bottom of the band, in general, where $\sin k \sim 0$. Another important difference is that in the electron case, a simple gauge transformation $\psi_{n}^{\prime}=(-1)^{n} \psi_{n}$ maps the system from $\left(K, \Omega, \lambda_{e}\right)$ to $\left(K+\pi,-\Omega,-\lambda_{e}\right)$. Thus, the transmission diagram for negative $\lambda_{e}$ is related to that of a positive $\lambda_{e}$, simply by an inversion of the $\Omega$ axis. No such transformation exists for Eq. (4). This means the transmission diagram in the $\left(\omega,\left|E_{t}\right|\right)$ plane will depend upon the sign of the nonlinearity. The superlattice structure modifies the dispersion in such a way that it breaks the symmetry between positive and negative nonlinearity.

Before presenting our numerical results, let us explain the procedure upon which these results are obtained. In the presence of nonlinearity, the transmission coefficient depends not only upon the frequency, but also upon the intensity of the incident wave. Indeed, the possibility of observing bistability and multistability, i.e., the same input yielding more than one possible output, is the key signature of the nonlinear phenomenon. ${ }^{4}$ Instead, a common approach is to solve the problem with a fixed output. Equation (4) can then be iterated backwards from the right to the left until we reach the outside of the nonlinear media on the input side. The electric field for $x<0$ is subsequently decomposed into the incident and reflected plane waves. The transmission coefficient is then evaluated from the definition given in Eq. (2).

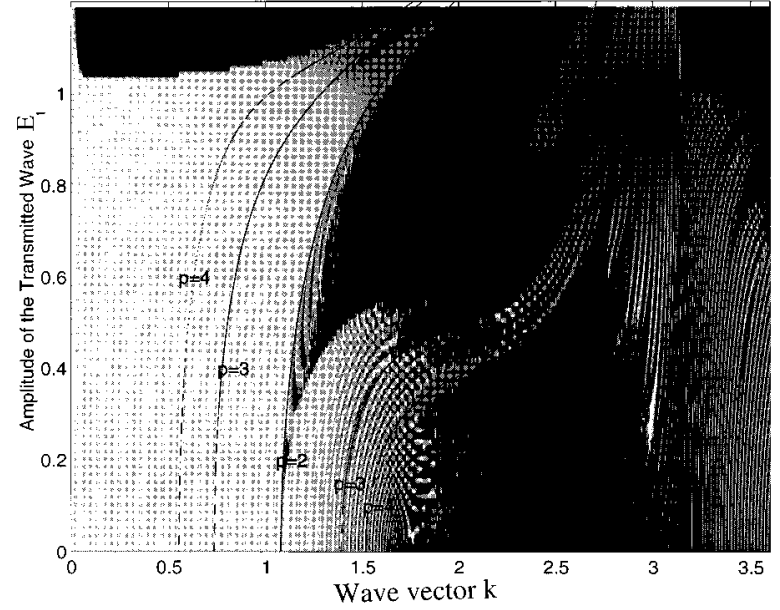

FIG. 2. The transmission diagram for a nonlinear superlattice with a negative Kerr coefficient showing the creation of passing regions in the stop bands of the linear systems and overall suppression of transmission in the lower frequency part. $N=80$ and $\alpha=1$. Lines are the trajectories of periodic orbits with the period indicated by the number. The lower periodic orbits bridge the gap completely for a large enough amplitude.

\section{GLOBAL TRANSMISSION DIAGRAM}

The results of our numerical calculations for an $N=80$ system with $\alpha=1.0$ are shown in Figs. 1 and 2, for positive $\lambda$ and negative $\lambda$, respectively. In these gray-scale plots of the transmission coefficient in the $\left(k,\left|E_{t}\right|\right)$ plane, the nonpassing regions appear as dark. We restrict the frequency to be in the first band, although similar structures are observed for all the bands. It is striking that the overall features, strongly modified by frequency as well as the field intensity, show dominant tongue structures and appear fractal-like. As the field strength increases, transmission stability diminishes and nonpassing regions appear and enlarge. Transmission stops beyond a frequency-dependent critical field strength. Other salient features include clear interference fringe in the transmission band, and stop-band widening for positive

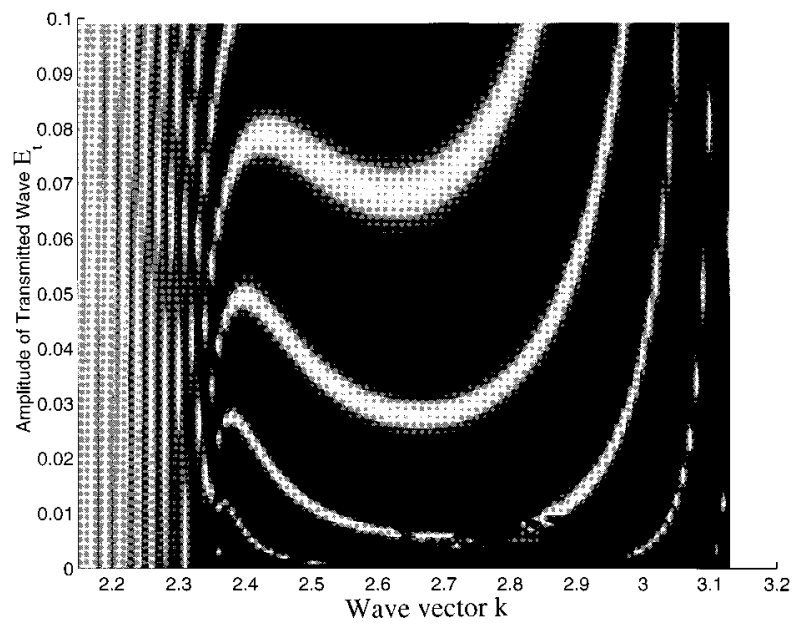

FIG. 3. The resonant transmission trajectories of single and multiple solitons in the first stop band of a nonlinear superlattice with a negative Kerr coefficient. $N=80$ and $\alpha=0.35$. Notice the extreme low field amplitude. 
Kerr-type nonlinearity, but narrowing for negative Kerr-type nonlinearity, as the output field strength increases. In fact, the gap seems to be completely bridged in the negative nonlinear system. Detailed examination of the transmission within the stop-band reveals well-defined transmission strips (Fig. 3) for negative nonlinearity only. At the end of this section, we shall show these are the trajectories of the resonant transmission via single soliton and soliton trains that were first observed by Chen and Mills. ${ }^{8}$

In the following subsections, we shall attempt to provide some theoretical understanding of these complex features and demonstrate that the main features can be understood from two important aspects of Eq. (4). First, each of the stable periodic orbits of the corresponding discrete nonlinear mapping possesses a stable basin and these basins form the dominant tongue and fractal-like structures. Second, the spectrum and the stability bounds of Eq. (4) control the overall shape of the transmission region. We shall also provide an intuitive mechanical analogy that will help understand the appearance of solitons and their trajectories in the $\left(k,\left|E_{t}\right|\right)$ plane.

\section{A. Nonlinear mapping}

Equation (4) can be viewed as a nonlinear discrete map as follows. We write the complex field $E$ in polar coordinates: $E_{n}=r_{n} e^{i \theta_{n}}$. The integral of motion for Eq. (4) becomes

$$
J=r_{n} r_{n-1} \sin \left(\Delta \theta_{n}\right),
$$

representing physically the conservation of current, where $\Delta \theta_{n}=\theta_{n}-\theta_{n-1}$. We introduce new dynamical variables: $u_{n}=r_{n}^{2}$ and $v_{n}=J \cot \left(\Delta \theta_{n}\right)$. We then obtain, from Eq. (4), the corresponding area-preserving nonlinear discrete map$\operatorname{ping} S:^{13}$

$$
S:\left\{\begin{array}{l}
u_{n-1}=\frac{1}{u_{n}}\left(v_{n}^{2}+J^{2}\right) \\
v_{n-1}=-v_{n}-u_{n-1}\left(\lambda_{e} u_{n-1}+\Omega\right)
\end{array},\right.
$$

where $\Omega=-(2 \cos k-\alpha k \sin k)$ and $\lambda_{e}=\lambda \alpha k \sin k$. The starting point of the mapping is $\left(u_{0}, v_{0}\right)=\left(\left|E_{t}\right|^{2},\left|E_{t}\right|^{2} \cos k\right)$, obtained from the physical boundary condition Eq. (1).

Starting from an arbitrary point in the $\left(k,\left|E_{t}\right|\right)$ plane, the mapping generated by Eq. (7), in general, will exhibit stable, unstable, or even chaotic trajectories. Stable and unstable orbits correspond to passing and nonpassing regions of the phase diagram, respectively. The unstable orbit diverges as $n$ decreases, producing an exponentially large value of the required input amplitude and equivalently, an exponentially small transmission for a finite input amplitude. Chaotic orbits, characterized by irregular field amplitude and phase as a function of $n$ and extreme sensitivities to initial conditions, are also found between the dominant tongues of transmission.

The initial condition that corresponds to stable periodic orbits can be obtained by iterating Eq. (7) from the starting values $\left(u_{0}, v_{0}\right)$ and demanding that the solution be periodic with period $p$. We have explicitly computed these curves for several lower period orbits ( $p$ up to 4; see Appendix) and the results are shown as solid and dashed lines in Figs. 1 and 2. These curves clearly trace the dominant tongues of the trans- mission diagram. Each of these stable periodic orbits supports a finite stability basin. The size of these basins diminishes as the intensity increases, and eventually vanishes completely at a critical value of intensity when the mapping turns from elliptic to hyperbolic. In fact, there are an infinite number of such periodic orbits starting at $k=2 \pi q / p$ on the $E_{t}=0$ axis. Because these orbits cannot cross each other and they terminate at different values of the critical intensity, the overall shape of the transmission diagram appears fractallike. The dominant tongue structure is an actual reflection of the strong stability of the lower-period orbits. The fractallike structure indicates the nonperturbative nature of the instability in nonlinear lattices. ${ }^{12}$

It is also interesting to see that the period-three and -four orbits actually cross from one side of the stop band to the other in the negative Kerr nonlinear coefficient media. The stability of the orbits apparently decreases when approaching the center of the stop band, leaving the bridging incomplete. We find when the frequency is in the gap, the trajectories of the periodic orbits are sensitive to the form of the initial condition in the mapping. This clearly indicates the importance of the physical boundary conditions. The use of vacuum as an embedding medium for the nonlinear system makes some of the phase space directly accessible to real experiments, the gap region in the negative Kerr case being a good example. This part of the phase space would not be relevant to the electron case if lattices (rather than a vacuum) were used outside the nonlinear system. In this case, one can study the region only by treating $\Omega$ and $k$ as independent variables. $^{13}$

\section{B. Spectra bounds}

A spectrum bound can be obtained in a mean field fashion by linearizing Eq. (4):

$$
\left|2 \cos k-\alpha k \sin k\left(1+\lambda\left|E_{t}\right|^{2}\right)\right| \leqslant 2 .
$$

This bound is placed on solutions with a uniform field amplitude throughout the structure. Although in our system no solutions of this type can exist due to interferences of the transmitting and the scattered waves by the surfaces at the two ends of the structure, Eq. (8) nevertheless provides a reasonable bound for stable transmission as shown in Fig. 4. This bound at $E_{t}=0$ coincides with the bound of a linear spectrum for a periodic Kronig-Penney $\delta$-function model. Equation (8) has simple physical solutions close to $k=m \pi$. For $\lambda>0$,

$$
\left|E_{t}\right|^{2} \leqslant \begin{cases}\frac{2}{\alpha \lambda k^{2}} & \text { for } k \sim 0 \\ \frac{2}{\alpha m \pi \lambda \delta} & \text { for } k=m \pi+\delta .\end{cases}
$$

For $\lambda<0$,

$$
\left|E_{t}\right|^{2} \leqslant \begin{cases}\frac{1+\alpha}{\alpha|\lambda|}\left(1-k^{2} / 6\right) & \text { for } k \sim 0 \\ \frac{1}{\lambda}\left(1+\frac{\delta}{m \pi \alpha}\right) & \text { for } k=m \pi+\delta .\end{cases}
$$



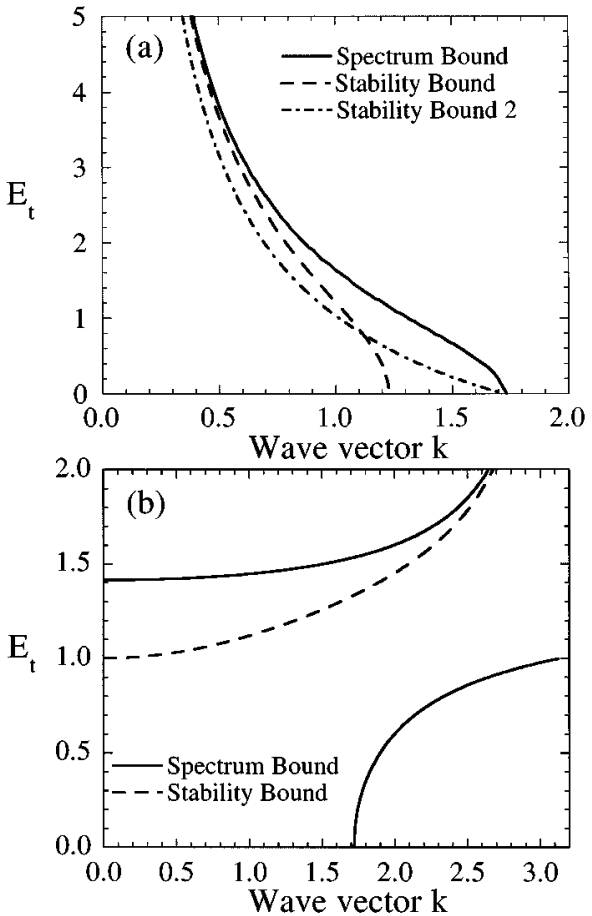

FIG. 4. The spectrum [Eq. (8)] and stability bounds [Eqs. (18) and (19)] in the first band for a nonlinear superlattice with a positive (a) and a negative (b) Kerr coefficient.

The spectrum bound analysis helps the understanding of the different responses at low frequencies between the positive and negative Kerr media. For positive Kerr media, the critical field amplitude to suppress transmission diverges at the bottom of the bands, while for negative Kerr media, it is finite. (See Figs. 1 and 2.) Physically, the overall suppression of transmitting regions for positive Kerr media is due to the increased contrast between the effective dielectric constant of the nonlinear and linear layers, and at the same time, decreased stability, as the field intensity increases. Since the nonlinearity is modified by the $\omega^{2}$ prefactor, the suppression is the weakest at low frequencies. In contrast, for negative nonlinear media, the bound is severe at low frequencies, while it is less below the bottom of the upper band. For negative Kerr media, increasing intensity has the effect of shrinking the stop band. The competition between the increased transmission bandwidth, on the one hand, and the decreased stability, on the other, makes the phase diagram quite complex. Notice in Fig. 2 the appearance of passing regions just below the upper band edge.

\section{Mechanical analogy and stability bound}

The previous subsection focused on the spectrum bound assuming a uniform amplitude (Bloch-like) solution that provides a rather reasonable qualitative understanding of the stability boundary found numerically. In actual calculations, the amplitude varies as one moves along the structure. In this section, we shall see that better bounds can be obtained if we examine the stability of these solutions under the assumption of a slowly varying field. Under such conditions, the problem of solving the coordinate-dependent field intensity can be mapped to the classical dynamics problem of a particle mov- ing in a centrifugal field. The coordinate $n$ is identified as the time variable $t$ and the field amplitude as the distance $r$. Within this mapping, the stability of the solution really means the particle will not go to infinity asymptotically.

Close to the "effective" band edge where the mean field solution is $e^{i K n}$, the mapping,

$$
F(t=n)= \begin{cases}E_{n} & \text { for } K \sim 2 m \pi \\ (-1)^{n} E_{n} & \text { for } K=(2 m+1) \pi,\end{cases}
$$

transforms Eq. (4) into the differential equation,

$$
\frac{d^{2} F}{d x^{2}}+c_{1} F+c_{2}|F|^{2} F=0,
$$

where $c_{1}=2(1 \mp \cos k) \pm \alpha k \sin k, c_{2}= \pm \alpha k \sin k$, and the upper (lower) sign is taken for $K=2 m \pi[K=(2 m+1) \pi]$.

Identifying $n$ with $t$ and $F$ with $r e^{i \theta}$, the above equation describes the motion of a unit mass particle in a potential

$$
V(r)=\frac{1}{2} c_{1} r^{2}+\frac{1}{4} c_{2} r^{4},
$$

with a conserved angular momentum

$$
L=r^{2} \frac{d \theta}{d t}=E_{t}^{2} \sin k
$$

and total energy

$$
E_{\mathrm{tot}}=\frac{1}{2}\left(\frac{d r}{d t}\right)^{2}+\frac{L^{2}}{2 r^{2}}+V(r) .
$$

The radial part of the motion is governed by

$$
V_{\mathrm{eff}}(r)=\frac{1}{2} c_{1} r^{2}+\frac{1}{4} c_{2} r^{4}+\frac{1}{2} \frac{L^{2}}{r^{2}} .
$$

This mechanical analogy is very appealing and intuitive. A similar analogy exists for a dispersive nonlinear film. ${ }^{4}$ When $c_{2}>0$, all solutions of Eq. (12) are stable. However, when $c_{2}<0$, the stability of the solution requires the particle bounded within the local minimum. Noticing the effective potential can be expressed as a function of intensity $I=|F|^{2}$ only,

$$
V_{\mathrm{eff}}(I)=\frac{1}{2} c_{1} I+\frac{1}{4} c_{2} I^{4}+\frac{I_{t}^{2} \sin ^{2} k}{2 I},
$$

the condition for the stability is then $d V_{\text {eff }} / d I<0$, i.e.,

$$
E_{t}^{2}<\frac{c_{1}-\sin ^{2} k}{\left|c_{2}\right|} \text {. }
$$

This bound is shown in Fig. 4, together with the spectrum bound for both the positive and negative Kerr media, obtained by solving Eq. (8). It is clear that the stability condition places a stronger bound than the spectrum bound and is generally in better agreement with the phase diagrams produced with numerical solutions of Eq. (4).

A second bound can be obtained by requiring that the particle be confined within the local minimum of $V(r)$ around $r=0$ (for $c_{1}>0$ and $c_{2}<0$ ). This condition is satis- 
fied when the total energy is less than the maximum of the potential barrier. Taking into account that $d r / d t=0$ at $t=0$, the condition is then

$$
V_{\text {eff }}\left(I_{t}\right) \leqslant \frac{c_{1}^{2}}{4\left|c_{2}\right|} .
$$

This bound (shown in Fig. 4 as stability bound 2) for positive Kerr media is in much better agreement with the numerical phase diagram, Fig. 1.

\section{Gap solitons}

The existence of stationary soliton solutions in the forbidden region of the linear system can also be understood through the mechanical analogy. A soliton is a localized excitation, which, strictly speaking, does not radiate in an infinite lattice. In the mechanical picture, this is possible only when the total angular momentum is close to zero. The particle starting with $r=0$ and moving in the potential will be reflected by the potential barrier at large $r$, and the time dependence of the coordinate $r$ produces precisely the envelop profile of a soliton. Solving the differential equation [Eq. (12)] with $E_{t}=0$ yields a single-soliton solution in an infinite lattice,

$$
F(n)=\sqrt{\frac{2}{-\lambda\left|c_{2}\right|}} \frac{1}{\xi} \operatorname{Sech}\left(\frac{n}{\xi}\right),
$$

where the extension of soliton $\xi=1 / \sqrt{\left|c_{1}\right|}$.

A simple physical picture can dispel the apparent mystery associated with the formation of the stationary soliton. The soliton forms only within the otherwise nonpassing region. In the forbidden region, the wave amplitude can be exponentially increasing or decreasing with $x$ and we reject the exponentially increasing solution, because it is not physical. However, in a nonlinear medium, the effective stop-band width depends upon the field intensity. When the field strength increases exponentially from a starting small value as one enters the nonlinear system from outside, the effective stop-band narrows and eventually closes. The field will then behave as if it is propagating within the pass band and starts to oscillate. But as soon as the field strength falls to a value such that a gap opens and the frequency is once again in the gap, the field begins to decrease exponentially. The overall shape of the field thus shows the localized structure of a soliton.

The analytical solution for the soliton profile compares well with the numerical results. An example is shown in Fig. 5 for a system exactly at resonance $(T=1)$ with $N=80$, $\alpha=0.35$ and a frequency just above the top of the first band and field strength $E_{t}=0.000035$. The phase of the soliton field (not shown) is almost a constant, except in the tail part where coupling between the soliton and the outside environment began to have an effect. We have checked that the soliton extension is independent of $E_{t}$, but the peak position of the soliton moves away from the center of the structure as $E_{t}$ departs from the resonant field amplitude.

Resonance transmission is achieved via the formation of solitons which, in turn, couple to the incident and output field. Resonance occurs when the tail of the soliton exactly matches the input and the output field at the surfaces with

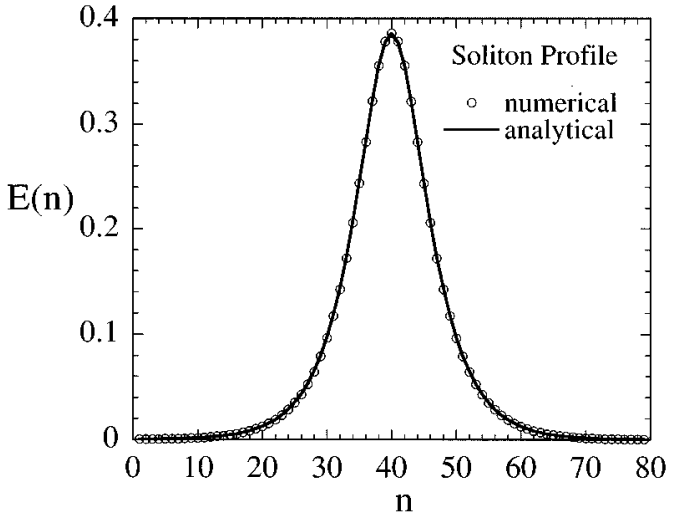

FIG. 5. Single-soliton profile in a nonlinear superlattice with a negative Kerr coefficient. $N=80$ and $\alpha=0.35$. The circles are the numerical values and the solid line is the analytical results, Eq. (20).

equal amplitude. When exactly $N_{s}$ solitons are confined within the structure, the resonant input (and output) field amplitude is given by a simple relation,

$$
E_{t}=\sqrt{\frac{2}{-\lambda\left|c_{2}\right|}} \frac{1}{\xi} \operatorname{Sech}\left(\frac{n}{2 N_{s} \xi}\right) .
$$

These trajectories are shown in Fig. 6 for $N_{s}$ up to 5 and should be compared with the numerical results shown in Fig. 3 . Given the approximate nature of the analytical solutions, the overall agreement with the numerical calculation is quite satisfactory.

Equation (11) clearly shows no soliton solution exists for positive Kerr media in the $\delta$-function model. In fact, this is a consequence of the $\delta$-function approximation. In this model, for positive nonlinearity and as the field strength increases, the upper band edge moves away from the frequency, while the lower band edge of the next band is fixed at $k=m \pi$. Not only does the stop band never close as the field strength increases, but it actually widens. As a result, gap solitons cannot form in the $\delta$ function model when the nonlinearity is positive. For nonlinear layers of finite width, soliton solutions do exist for both positive and negative Kerr media, because both band edges shift with intensity. This is in agreement with the conclusion of Chen and Mills, ${ }^{8}$ who

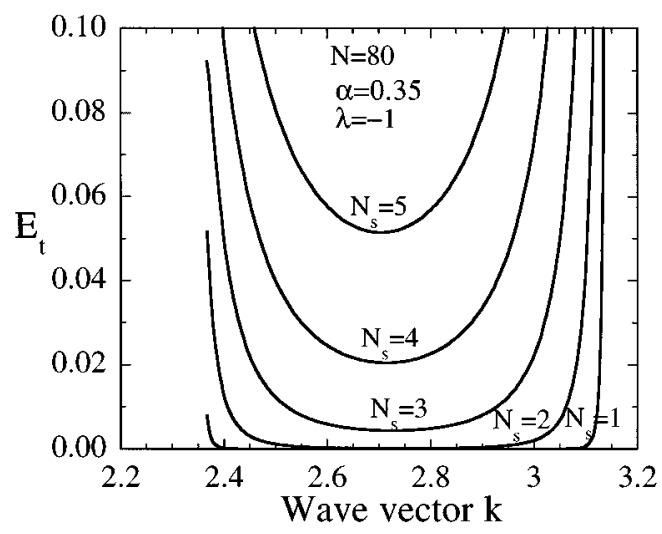

FIG. 6. Multiple-soliton trajectories from the analytical prediction [Eq. (21)] for the same system of Fig. 3. 


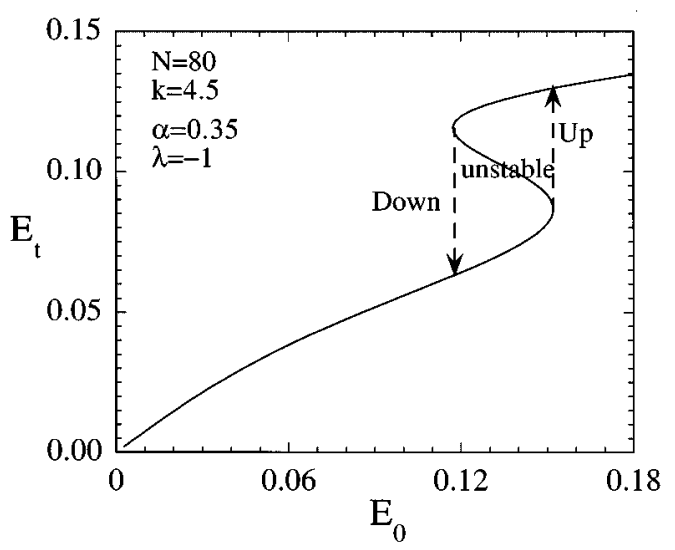

FIG. 7. Transmission curve of a typical bistable system. Notice regions with three output solutions for the same input. The part with a negative slope is unstable. The switch up and switch down occur as indicated by the arrows.

found solitons at the upper edge for positive Kerr media and at the lower edge for negative media.

Finally, we note that other types of nonstationary solitons may exist in the gap of a Bragg reflector. These solitons can propagate with a velocity between zero and the average speed of light in the medium and exhibit relativistic behavior. $^{9,15,16}$

\section{BISTABILITY AND SWITCHING THRESHOLD}

In this section, we focus on the bistability and multistability induced by the periodic modulation of nonlinear media. Bistability is said to occur when the system has two output states for the same value of input over some range of input values. Which state of these possible outputs the system follows depends upon the detail history and dynamics of the input. As an example, the input-output characteristics of an $N=80$ system with the frequency inside the second transmission band show the generic characteristics for an optical bistable system (Fig. 7). This curve is obtained by varying $E_{t}$. The part of the curve with a negative slope is unstable, and the arrows indicate the actual path that the system takes in response to an increasing or decreasing input field. The hysteresis loop is a signature of the bistable response. An important quantity is the switch-up and -down intensity that measures the energy it takes to operate the switch. To achieve low switching threshold in application, both switch-up and switch-down intensity are required to be low.

In the nonlinear wave propagation problem that we are considering, bistability occurs quite generally when the transmission coefficient is strongly modified by the field intensity. We classify the bistability into three categories according to the way they occur, (1) bistability within the transmitting regions, (2) bistability between the tongues, and (3) bistability via soliton formation (only for $\lambda<0$ in our model). Two typical responses are shown in Figs. 8 and 9, respectively, for frequencies inside and outside the transmission band.

The bistability within the transmitting band operates exactly with the same principle as the nonlinear Fabry-Perot devices. ${ }^{4}$ The modulation of the dielectric constant by the field inside the nonlinear medium produces an extra phase
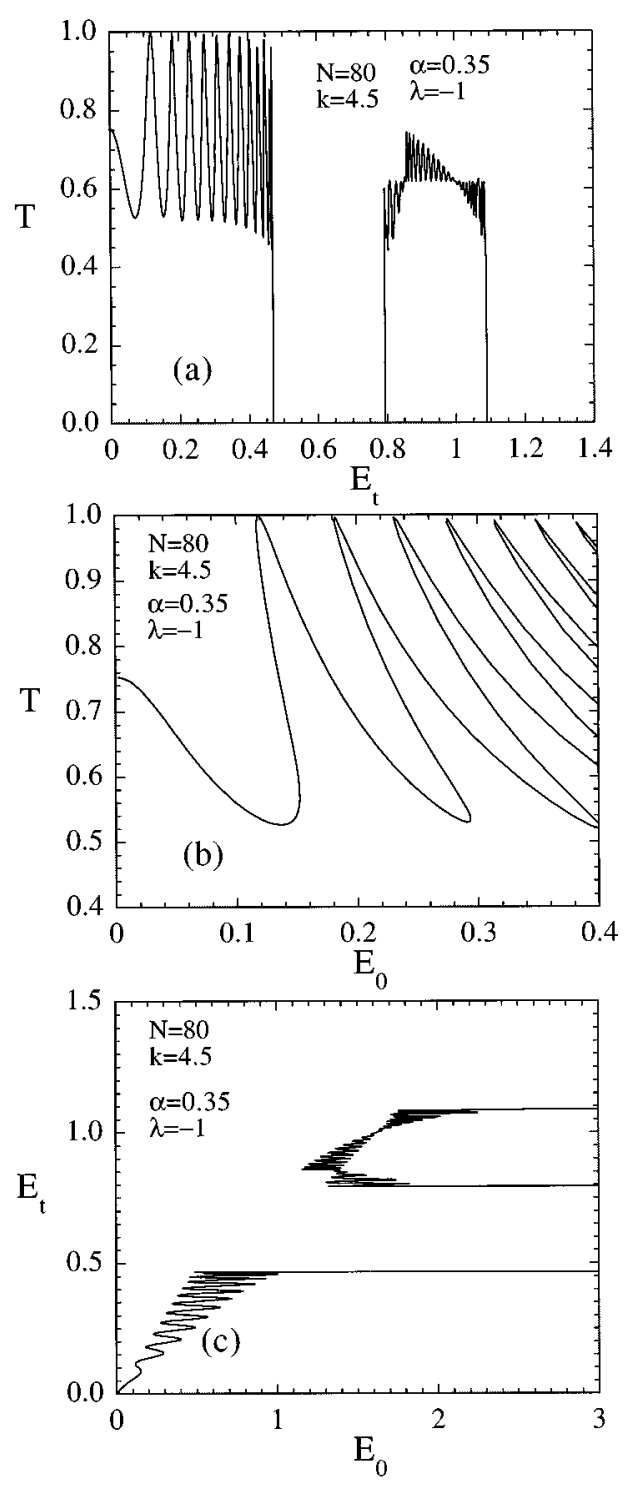

FIG. 8. Typical transmission characteristics when the frequency is inside the transmission band. (a) Transmission coefficient vs output field amplitude; (b) transmission coefficient vs input field amplitude; and (c) output field amplitude vs input field amplitude. Notice the extreme optical limiting as evidenced by the plateau structure in (c).

shift, which changes the interference pattern and hence the transmission coefficient. To accumulate over the entire sample a phase shift of the order of $\pi$ required for switching, both the field strength and the length of the sample must be large. This is illustrated in Fig. 7 and in the low field part of Figs. 8(a)-8(c). The switch-up and switch-down intensities are generally of the same order of magnitude. The switching intensity varies as the inverse of the total length of the structure if absorption is neglected.

The second class of bistability occurs when the field intensity crosses from one tongue to another (see Figs. 1 and 2 ). Figure 8 shows the modulation of the transmission coefficient when the output [Fig. 8(a)] or the input [Fig. 8(b)] field amplitude is increased. Notice the existence of a nonpassing region $(T=0)$ in the middle in Fig. 8(a). The two output states are characterized by being at different transmis- 

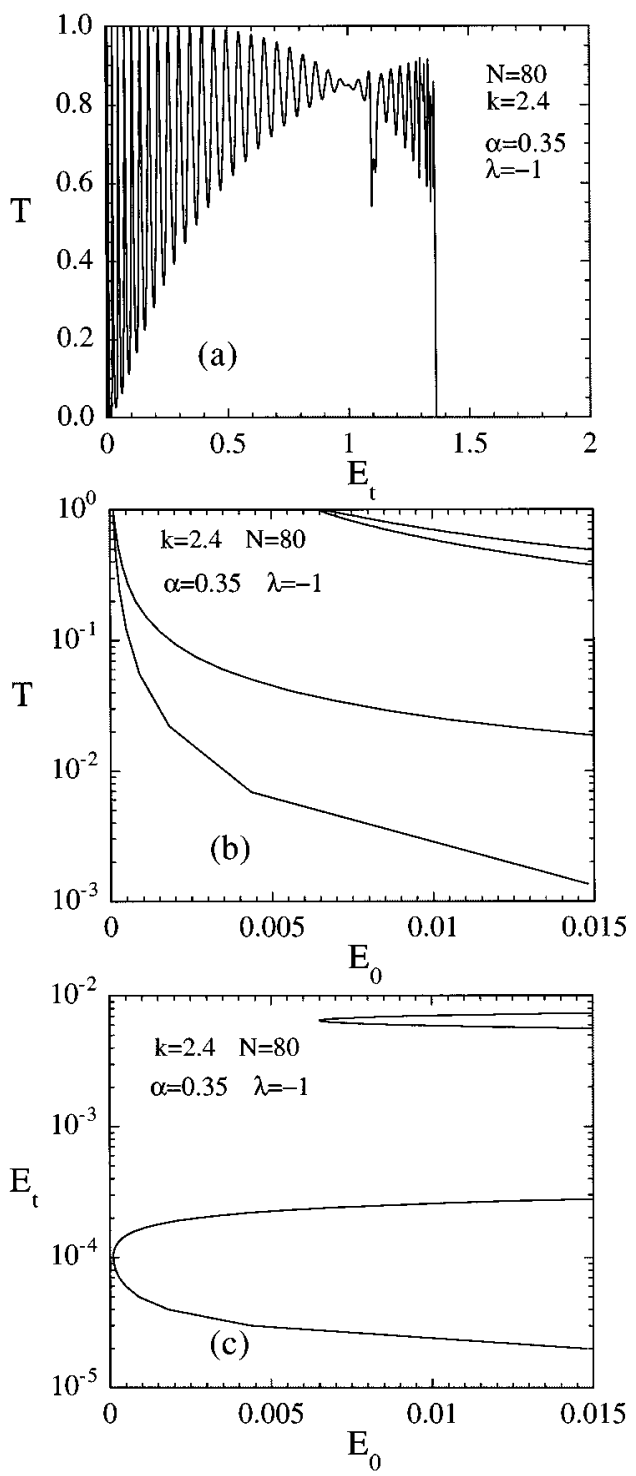

FIG. 9. Typical transmission characteristics when the frequency is in the stop band. (a) Transmission coefficient vs output field amplitude; (b) transmission coefficient vs input field amplitude; and (c) output field amplitude vs input field amplitude. Notice the logarithmic scale in the $y$ axis in (b) and (c).

sion tongues. Because the crossover between the tongues occurs only when the nonlinearity (or the scaled field strength $E$ ) is of the order of one, the switch-down intensity is much larger as compared with that of the first class. The switchdown intensity cannot be reduced by increasing the size of the structure. Moreover, since the instability in the nonpassing region is strongest with a diverging rate of $\exp \left(3^{N}\right)$, the plot of $E_{t}$ vs $E_{0}$ shows plateau structures [Fig. 8(c)]. Similar behavior was observed in a previous study of the electron transmission in nonlinear lattices. ${ }^{12}$ Therefore, the switch-up intensity can be orders of magnitude (in fact, exponentially) larger than the switch-down intensity.

The third class of bistability occurs via gap-soliton transmission when the frequency is in the stop band of the linear system. The transmission states are characterized by the number of solitons contained in the resonant state. Figure 9(a) shows the transmission coefficient as a function of the output field amplitude $E_{t}$. The resonance transmission at low field amplitude is a result of soliton and soliton-train formation. The single-soliton and two-soliton transmission characteristics are shown in Figs. 9(b) and (c). Notice the extremely small values of the switch-down field. In fact, the switchdown field decreases exponentially with the system size and is given by Eq. (20). The reduced threshold is due to the concentration of energy on localized areas. This attribute is extremely advantageous for highly nonlinear systems with absorption, for example, semiconductor nonlinear materials in the vicinity of the electronic band edge. Unfortunately, at the switch up between the different soliton transmission states, the transmission is extremely low. Hence, the switch-up field is very high [not shown in Fig. 9(c)].

All the experiments on distributed feedback structures have been done utilizing the first class of bistability. As we remarked before, the second class bistability requires large nonlinearity and are not suitable for switches. However, it can be used very well for optical limiting. The third class bistability apparently has the promising advantage of an extremely low switch-down threshold. However, there are several difficulties with this type of bistability. The nonlinear effect in most of the materials saturates at high intensity. This may hinder the formation of gap solitons, because their existence still requires a large nonlinear effect at the peak positions. Moreover, effective coupling between the incident wave and the gap soliton could be a problem, due to the sensitivity to the precise value of the input to achieve resonance, as the size of the system is necessarily large for low threshold applications.

\section{CONCLUSIONS}

In conclusion, we have investigated the propagation of EM waves in a superlattice of layered structure with an intensity-dependent dielectric constant. The transmission diagrams in the frequency-amplitude plane exhibit interesting and complex tongue structures, due to the increasing instability induced by the the periodic modulation of the intensity. For positive Kerr media, the transmission is severely suppressed away from the bottom of the band. For negative Kerr media, transmission within the stop band is possible with increasing intensity. Moreover, soliton solutions exist within the stop band, and through coupling, resonance transmission can be achieved. These complex responses are explained by analyzing the spectrum bound and stable periodic orbits of the corresponding nonlinear mapping. Utilization of solitons to confine energies within small localized areas can reduce the switching threshold to a value that is reachable within the currently available radiation intensity. However, several difficulties must be overcome before one can build switches operating under soliton resonance transmission conditions, as discussed in Sec. IV.

\section{ACKNOWLEDGMENTS}

Ames Laboratory is operated for the U. S. Department of Energy by Iowa State University under Contract No. W-7405-ENG-82. This work was supported by the Director of Energy Research, Office of Basic Energy Science, and Advanced Energy Projects. 


\section{APPENDIX A}

In this appendix, we give the equations satisfied by the lower periodic orbits of the mapping Eq. (5) with the starting point $\left(u_{0}, v_{0}\right)=\left(\left|E_{t}\right|^{2},\left|E_{t}\right|^{2} \cos k\right)$. We introduce new variables $\Omega=-(2 \cos k-\alpha k \sin k), \quad \lambda_{e}=\lambda \alpha k \sin k, \quad$ and $x$ $\equiv \lambda_{e}\left|E_{t}\right|^{2}$. The trajectories for the periodic orbits satisfy the following equations.

For the period-one orbit,

$$
x=-(2+\Omega) .
$$

For the period-two orbit,

$$
x=-\Omega \text {. }
$$

For the period-three orbit,

$$
\begin{gathered}
x^{5}+4 a x^{4}+\left(4 a^{2}+2 b\right) x^{3}+(4 a b+\Omega) x^{2}+\left(2 a \Omega+b^{2}-2\right) x \\
+(2 a+b) x=0 .
\end{gathered}
$$

For the period-four orbit,

$$
f^{2}-\left(x f^{2}+\Omega f-x-a\right)^{2}-\sin ^{2} k=0,
$$

where $a \equiv \Omega+\cos k, b \equiv a^{2}+\sin ^{2} k$, and $f(x) \equiv x^{2}+2 a x+b$.
${ }^{1}$ Photonic Band Gaps and Localization, edited by C. M. Soukoulis (Plenum, New York, 1993); Photonic Band Gap Materials, edited by C. M. Soukoulis (Kluwer Academic, Dordrecht, 1996).

${ }^{2}$ A. Hasegawa and F. D. Tappert, Appl. Phys. Lett. 23, 142 (1973).

${ }^{3}$ H. M. Gibbs, S. L. McCall, and T. N. C. Venkatesant, Phys. Rev. Lett. 36, 1135 (1976).

${ }^{4}$ J. H. Marburger and F. S. Felder, Phys. Rev. A 17, 335 (1978).

${ }^{5}$ H. M. Gibbs, Optical Bistability: Controlling Light with Light (Academic, Orlando, FL, 1985).

${ }^{6}$ H. G. Winful, J. H. Marburger, and E. Garmire, Appl. Phys. Lett. 35, 379 (1979).

${ }^{7}$ J. He, M. Cada, M.-A. Dupertuis, D. Martin, F. Morier-Genoud, C. Rolland, and A. J. SpringThorpe, Appl. Phys. Lett. 63, 866 (1993); B. Acklin, M. Cada, J. He, and M.-A. Dupertuis, ibid., 63, 2177 (1993).
${ }^{8}$ Wei Chen and D. L. Mills, Phys. Rev. B 36, 6269 (1987); Phys. Rev. Lett. 58, 160 (1987).

${ }^{9}$ C. Martine de Sterke and J. E. Sipe, in Progress in Optics, edited by E. Wolf (Elsevier, Amsterdam, 1994), Vol. 33.

${ }^{10}$ S. John and N. Akozbek, Phys. Rev. Lett. 71, 1168 (1993).

${ }^{11}$ M. Scalora, J. P. Dowling, C. M. Bowden, and M. J. Bloemer, Phys. Rev. Lett. 73, 1368 (1994).

${ }^{12}$ F. Delyon, Y. Levy, and B. Souillard, Phys. Rev. Lett. 57, 2010 (1986).

${ }^{13}$ Yi Wan and C. M. Soukoulis, Phys. Rev. B 41, 800 (1990).

${ }^{14}$ D. Hennig, H. Gabriel, G. P. Tsironis, and M. Molina, Appl. Phys. Lett. 64, 2934 (1994).

${ }^{15}$ D. N. Christodoulides and R. I. Joseph, Phys. Rev. Lett. 62, 1746 (1989).

${ }^{16}$ A. B. Aceves and S. Wabnitz, Phys. Lett. A 141, 37 (1989). 